\title{
Rationale for new model development
}

Dr. Bruce Hampton, an orthopedic surgeon, was a high-energy researcher who always had a new project on the horizon. Hampton had been using rabbits as his animal model for nearly two decades. The cost of purchasing and housing rabbits was increasing every year, whereas his federal grant funding was moving in the opposite direction. To compensate for this discrepancy, Hampton decided to try to develop a rat model of the orthopedic condition he was studying. The process he proposed to the Great Eastern University IACUC was to use five rats in a pilot study to determine whether the surgical modifications he made on rabbit femurs could be duplicated and remain effective in the smaller animal model. If the surgical procedures proved to be effective, he then would move ahead with the various treatment methods that he was studying.

At the next full committee meeting of the IACUC, Hampton's protocol was presented by Dr. Alex Burke. Burke pointed out some minor inconsistencies, but overall, he strongly supported Hampton's plan for developing a new, efficacious and much less expensive animal model. Only one IACUC member questioned the proposed new model, asking why five rats had to be subjected to a major survival surgical procedure when there already was a perfectly acceptable model for Hampton to use. The response from Burke was that without the new model, Hampton might not have sufficient funds to continue his research, and that in any case, a new animal model is always a welcome addition to the research armamentarium.

What is your opinion? Should the IACUC consider the cost of using rabbits as a factor in its discussion of Hampton's protocol? Should the rationale for the development of the rat model be based on its possible need to sustain Hampton's research, or is the general concept of having a second model available a sufficient reason?

\section{RESPONSE}

\section{Not so fast}

\section{Patricia A. Preisig, PhD \& Kelly A. Fusco, CPIA}

The task at hand for the Great Eastern University IACUC was to evaluate a pilot study. Although research cost and career sustainability are valid concerns for investigators, neither should be the sole or a major factor influencing the IACUC's discussion. Assuming that the rabbit model was both scientifically justified and appropriate for Hampton's studies during the preceding two decades, the IACUC's deliberation of the pilot study request seems to be incomplete.

Irrespective of Hampton's incentive for developing a new model, the IACUC must require him to present a logical rationale for doing so, even for a pilot study ${ }^{1-3}$. Pilot studies are often discrete feasibility studies that are limited in animal number. Nevertheless, a proposed pilot study must be accompanied by a scientific rationale and a confirmation that it does not unnecessarily duplicate known work. Hampton's request should have included the intellectual basis for proposing that development of an alternative model is a logical approach to advancing the project in the face of limited resources and an adequate justification for the number of animals proposed to be included in the pilot study. The rationale could include physiological, anatomical or mechanical features of rodents; advantages and disadvantages of a rodent model; or the homologous and analogous characteristics of the relevant tissues and systems in rodents compared with rabbits, but it must provide enough information for the IACUC to determine whether the pilot study is justified. If the focus of the discussion was on the factors of cost and research program sustainability, then the IACUC's deliberations were not in keeping with its responsibilities.

If the pilot study is approved and Hampton later submits another protocol proposing to use additional animals to advance the model, then that request should include additional justification based on the pilot study results, with some indication as to whether the surgical manipulation of the femur could be duplicated and remain effective in the smaller animal. Such justification could include the potential for the rodent model to meet some or all of the project goals, including speciesspecific requirements; a discussion of the impact of combining data from different species on the interpretation, validity and quality of the data; and a description of the model's value toward advancing scientific knowledge and to society in the context of the principles of the $3 \mathrm{Rs}^{4}$.

1. Public Health Service. Policy on Humane Care and Use of Laboratory Animals (US Department of Health and Human Services, Washington, DC, 1986; amended 2002).

2. Animal Welfare Act and Animal Welfare Regulations. Part 2, Subpart C, Research Facilities.

3. Institute for Laboratory Animal Research. Guide for the Care and Use of Laboratory Animals 8th edn. (National Academies Press, Washington, DC, 2011).

4. Russell, W.M.S. \& Burch, R.L. The Principles of Humane Experimental Technique (Methuen, London, 1959).

Preisig is a Professor of Medicine and Cellular \& Molecular Physiology and the IACUC Chair, and Fusco is the Assistant Director for Compliance in the IACUC Office at Yale University, New Haven, CT.

\section{RESPONSE}

\section{The economy of research}

\author{
Charles Cates, DVM, \\ Joanne Zahorski-Reeves, DVM, PhD, \\ DACLAM, Marcelo Couto, DVM, PhD, \\ DACLAM \& Michael Campagna, DVM
}

Laboratory animal regulations are thoroughly silent on the financial evaluation of research protocols. The Animal Welfare Act $^{1}$ nowhere 